\title{
Ameliorative Role of Vitamin D on Prenatal and Postnatal Exposure of Monosodium Glutamate Induced Steatohepatitis in Rat Pups
}

\author{
Nandan Padmanabha' ${ }^{1}$ Nirupama Muralimunglimane ${ }^{2}$, Nayanatara Arun Kumar ${ }^{3 *}$, Bhagyalakshmi Kodavanji ${ }^{3}$, \\ Jyoti Ramnath Kini'², Roopesh Poojary ${ }^{4}$
}

\section{Nandan Padmanabha', Nirupama Muralimungli- mane $^{2}$, Nayanatara Arun Kumar $^{3 *}$, Bhagyalakshmi Kodavanji ${ }^{3}$, Jyoti Ramnath $\mathrm{Kini}^{2}$, Roopesh Poojary ${ }^{4}$}

\begin{abstract}
'Medical Student, Final MBBS-1, Kasturba Medical College Mangalore, Manipal Academy of Higher Education (MAHE), Karnataka, INDIA. ${ }^{2}$ Department of Pathology, Kasturba Medical College, Manipal Academy of Higher Education (MAHE), Mangalore, Karnataka, INDIA.

${ }^{3}$ Department of Physiology, Kasturba Medical College, Manipal Academy of Higher Education (MAHE), Mangalore, Karnataka, INDIA.

${ }^{4}$ Senior Research Fellow, Department of Physiology. Kasturba Medical College, Mangalore, Manipal Academy of Higher Education (MAHE), Karnataka INDIA.
\end{abstract}

Correspondence

Dr. Nayanatara Arun Kumar (M. Sc, Ph.D)

Associate Professor in Physiology Kasturba Medical College, Manipal Academy of Higher Education (MAHE), Bejai

Karnataka, INDIA.

Phone No: 9845551070

Fax: 918242428183

E mail Id: nayanaarun@hotmail.com

History

- Submission Date: 25-10-2017;

- Review completed: 18-11-2017

- Accepted Date: 27-12-2017

DOI : 10.5530/pj.2018.2.62

Article Available online

http://www.phcogj.com/v10/i2

Copyright

(c) 2018 Phcog.Net. This is an openaccess article distributed under the terms of the Creative Commons Attribution 4.0 International license.

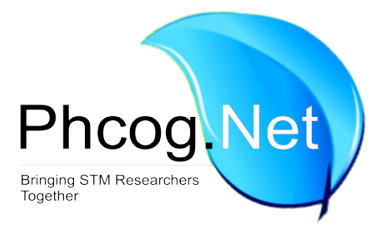

\begin{abstract}
Background: Monosodium glutamate (MSG) is a widely used flavor enhancer has been linked to obesity and metabolic syndrome, including progressive liver disease. Nonalcoholic Fatty Liver Disease (NAFLD) is the most common hepatic disorder with an etiology related to changes in diet and lifestyle. Prenatal and postnatal exposure to MSG been shown to affect developing tissues in growing animals. Increased Risk of Non-alcoholic Steatohepatitis has been associated with Vitamin D deficiency. The present study was aimed to investigate the ameliorative effect of vitamin D on MSG induced animal models of steatohepatitis in neonatal rats. Materials and Methods: Eighteen nulliparous female wistar rats were randomly divided into three groups ( $n=6 /$ group). Group-I received a daily oral dose of $5 \mathrm{~g} / \mathrm{kg}$ body weight of MSG. Group-II received the same dose of MSG along with calcitriol $(0.2 \mu \mathrm{g} / \mathrm{kg} \mathrm{BW})$. Group-III was treated with saline served as the control. The rats could mate, and treatment was given for the entire period of gestation and thirty days thereafter, during lactation. The histological changes in the liver was observed. Results: Pan-lobular microvesicular steatosis, lobular inflammation and ballooning of hepatocytes was observed in the MSG-treated group. These histotoxic changes were ameliorated in the vitamin D treated group. Conclusion: Vitamin D might be beneficial in the protection of the pre-and postnatal exposed MSG induced steatohepatitis. Further, induction of steatohepatitis in a shorter period could also make it an ideal study model of non-alcoholic steatohepatitis.
\end{abstract}

Key words: Vitamin D, Glutamate, Steatohepatisis, Gestation, MSG.

\section{INTRODUCTION}

Monosodium glutamate (MSG) is the sodium salt of the non-essential amino acid glutamic acid, commonly used as a taste enhancer in fast foods and Chinese restaurants. Its consumption has increased globally in recent years, with recent estimations of the current average daily intake believed to be up to $10 \mathrm{~g} /$ day/ person. ${ }^{1}$ However, the safety of this compound is not established and has been linked to metabolic and neurological complications. Several hypotheses have been put forward for the pathogenesis of this toxicity. ${ }^{2}$ Most plausible hypothesis involves induction of insulin resistance and central obesity brought about by ablation of the arcuate nucleus, the effect being more pronounced in neonates owing to immature blood-brain barrier. ${ }^{3}$ It involves glutamate-induced degeneration of regions that regulate feeding and satiety. ${ }^{4}$ The insulin resistance along with increased food intake could thus initiate the process of steatosis progressing to steatohepatitis. It is believed that high levels of MSG in the diet of a pregnant woman pregnant women is causative for pathological changes in the developing tissues in the growing neonates. Similarity and analogy in the genomes of the rodents and of the humans places these animal models as an important tool in the study of conditions that affect us and that can be simulated in rats.

The active form of Vitamin D, 1, 25-dihydroxy cholecalciferol $\left[1,25(\mathrm{OH})_{2} \mathrm{D}\right]$ is primarily involved in calcium and phosphate metabolism. Extensive exploration of its possible physiological functions over the past few years have proven its role in immune modulation, inflammation regulation, cell differentiation, and fibrosis, most of which could majorly affect the development and progression of NAFLD. Literature survey shows the positive correlation between NAFLD and vitamin D deficiency. ${ }^{5,6}$ The present study was aimed to investigate the ameliorative effect of vitamin D on MSG induced animal models of steatohepatitis in neonatal

\section{MATERIALS AND METHODS}

\section{Experimental animals}

Institutional Animal Ethics approval was obtained. A total of 18 adult Wistar rats (weighing 170-200g) were obtained from the Institutional Animal House,

Cite this article: Padmanabha N, Nirupama M, Kumar NA, Bhagyalakshmi K, Kini JR, Poojary R. Ameliorative Role of Vitamin D on Prenatal and Postnatal Exposure of Monosodium Glutamate Induced Steatohepatitis in Rat Pups. Pharmacog J. 2018;10(2):371-5. 
were acclimatized for five days in the experimental lab before the experiment. Lab conditions of 12:12h light: dark cycles and at room temperature $26 \pm 1^{\circ} \mathrm{C}$ were maintained. They were contained in individual cages with paddy husk as bedding material. The animals were provided with regular pellet diet and water free access to water. All experimental procedures followed strict guidelines as provided in the "Guide for the Care and Use of Laboratory Animals".

\section{Animal Grouping}

The rats were randomly divided into three groups (n=6/group). Group-I received a daily oral dose of $5 \mathrm{~g} / \mathrm{kg}$ body weight of MSG. Group-II received the same dose of MSG along with calcitriol $(0.2 \mu \mathrm{g} / \mathrm{kg}$ BW). Group III served as a control group which was treated with normal saline orally. The rats were treated during the entire period of gestation and thirty days thereafter, during lactation. Pups from the litters (6/group) were randomly selected from each group on the thirtieth day and sacrificed using ketamine and liver tissue was dissected for histological analysis. Figure 1.

\section{Calculation of monosodium glutamate dosage}

MSG was obtained as crystals of $99.9 \%$ purity manufactured by Life Science Laboratories Pvt Ltd., Chennai. A stock solution was prepared by dissolving $250 \mathrm{~g}$ of MSG granules in $500 \mathrm{~mL}$ saline. Dosages were calculated as per the body weight of animals ( $5 \mathrm{~g} / \mathrm{kg}$ bodyweight) and it was administered orally through a garage.

\section{Calculation of calcitriol dosage}

Calcitriol (1,25-dihydroxycholecaliferol) ampoules of $1 \mathrm{mg} / \mathrm{mL}$ manufactured by Akums drugs and pharmaceuticals Ltd., Haridwar India were mixed in normal saline to result in a concentration of $0.06 \mathrm{mg} / \mathrm{mL}$. Dosages were calculated as per the body weight of the animals. Cacitriol was administered through an oral gavage $(0.2 \mathrm{mg} / \mathrm{kg}$ bodyweight).

\section{Histological evaluation}

The liver was preserved in in $10 \%$ buffered formaldehyde for histologic examination of liver damage. After tissue fixation, the liver samples were taken and embedded in paraffin wax and stained with haematoxylin and eosin solution. The samples were coded to avoid bias and were evaluated and graded.

\section{NASH Scoring}

Morphological changes in the liver were assessed using NASH (non-alcoholic steatohepatitis) activity score, which included histological features which has been defined as the un-weighted sum of scores for steatosis (0-3), lobular inflammation (0-3) and ballooning (0-2). According to NASH scores $\geq 5$ are diagnosed as NASH, and cases with the score $1<3$ were not taken into consideration as NASH.?

\section{Statistical analysis}

The data were expressed as Mean \pm SD from 6 animals in each group. The differences between the groups were compared for statistical significance by the Student's $t$-test with the level of significance set at $\mathrm{p}<0.05$.

\section{RESULTS}

All samples in the control group (Group III) showed normal liver histology. The average NASH score was zero and none of the sections showed acidophil bodies, pigmented macrophages, mega mitochondria, glycogenated nuclei [Figure 2]. All the sections in Group I showed grade-3 steatosis $(>66 \%$ ) [Figure 3] and five of Group II showed grade-2 steatosis (33-66\%) [Figure 4] and one section of Group II showed grade-1 steatosis $(<33 \%)$ steatosis. The difference observed between the groups in the

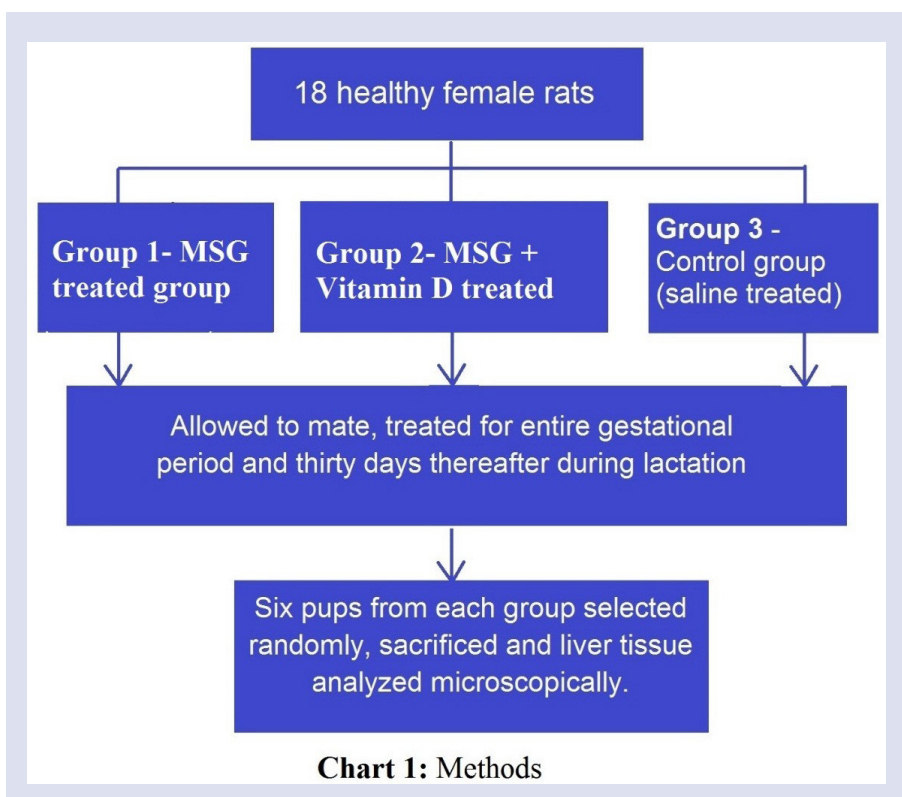

Figure 1: Animal grouping.

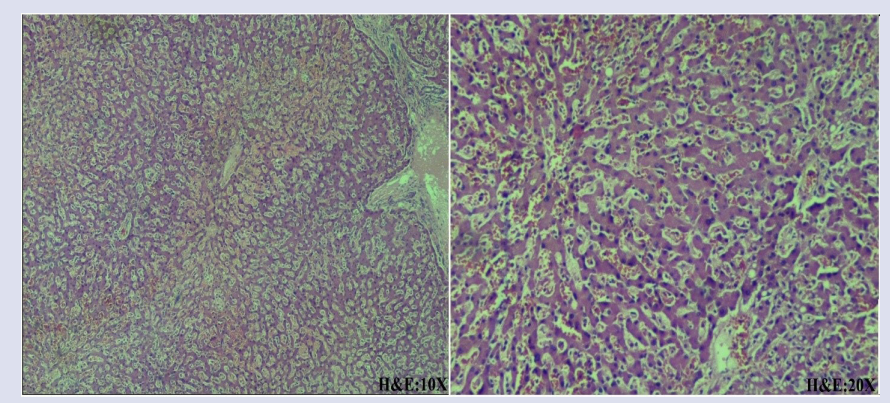

Figure 2: Liver parenchyma as seen in the control group.

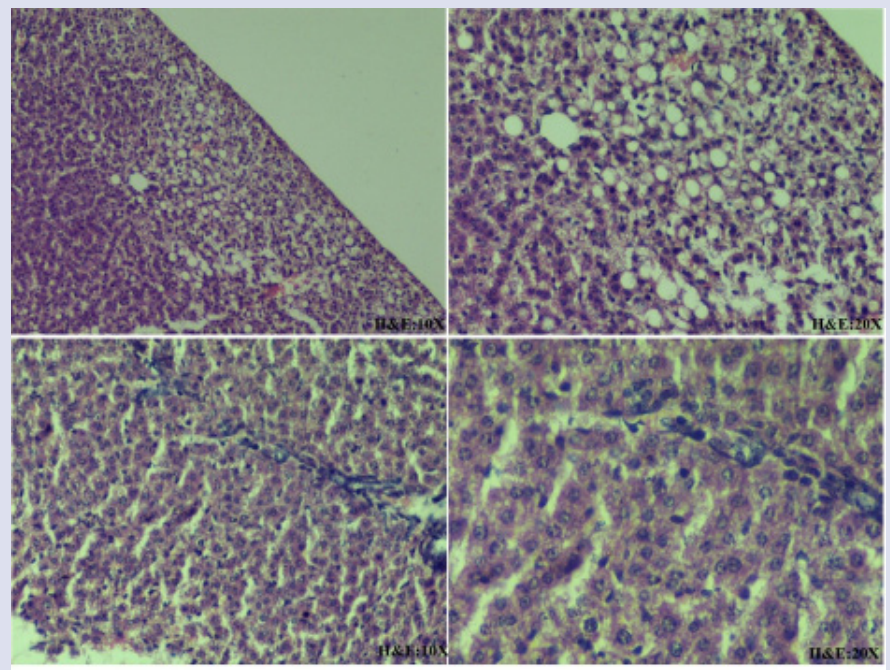

Figure 3: Microvesicular, macrovesicular steatosis and fatty change as seen in the MSG-treated group (top row). Perisinusoidal fibrosis was seen in the MSG-treated group (bottom row). 


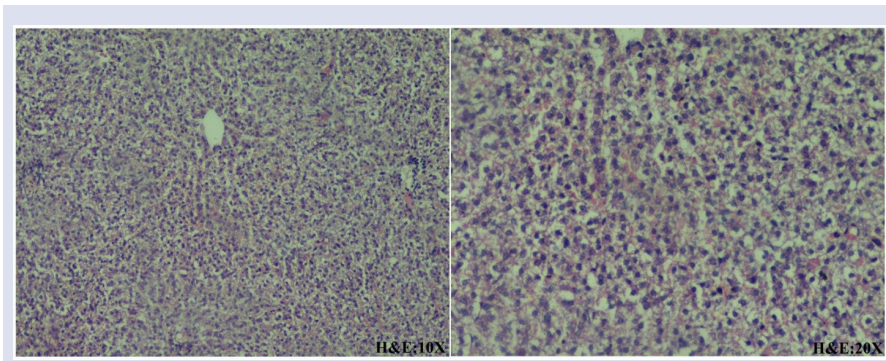

Figure 4: Liver parenchyma as seen in the MSG+ Vitamin D treated group showing grade 2 steatosis.

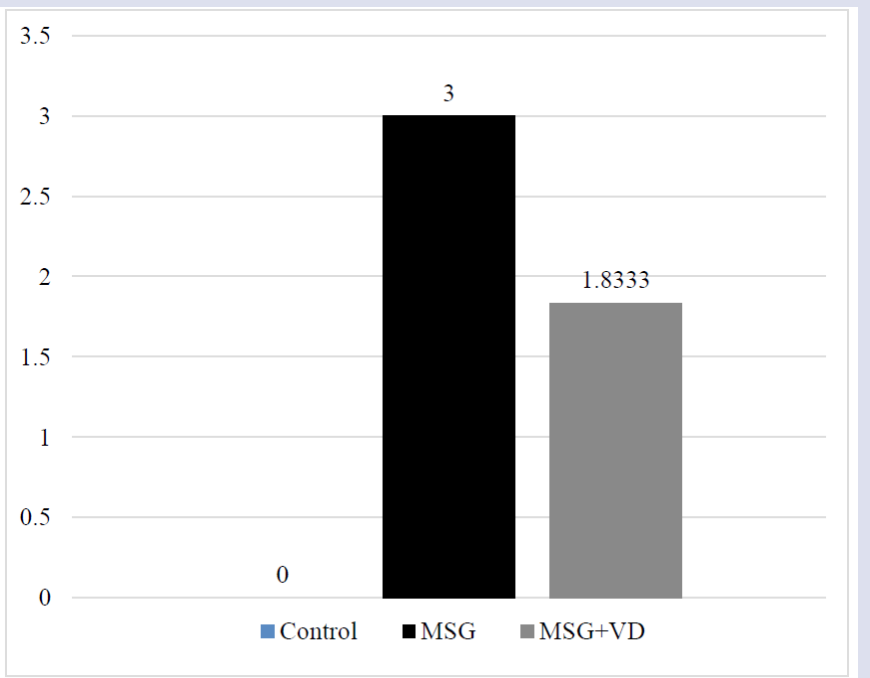

Figure 5: Average Steatosis Grade.

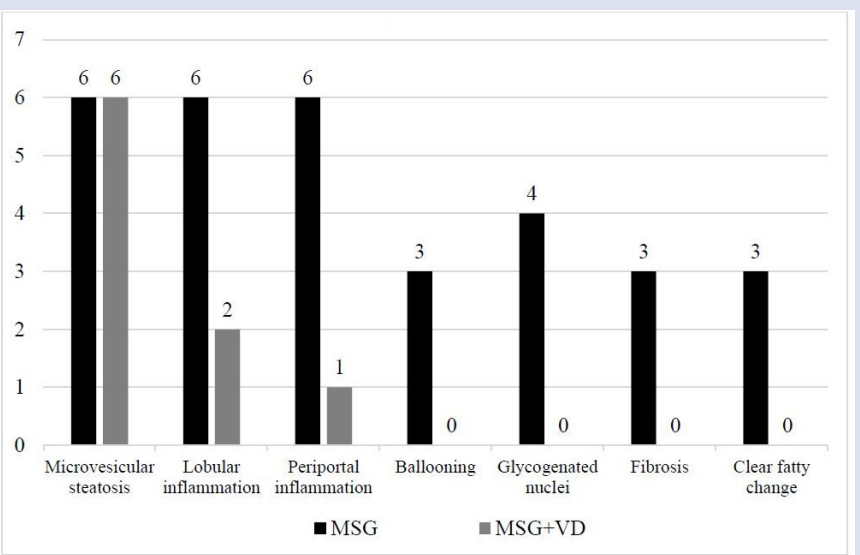

Figure 6: Comparision of liver Histologic changes in the Group I (MSG) and Group II (MSG+Vit D).

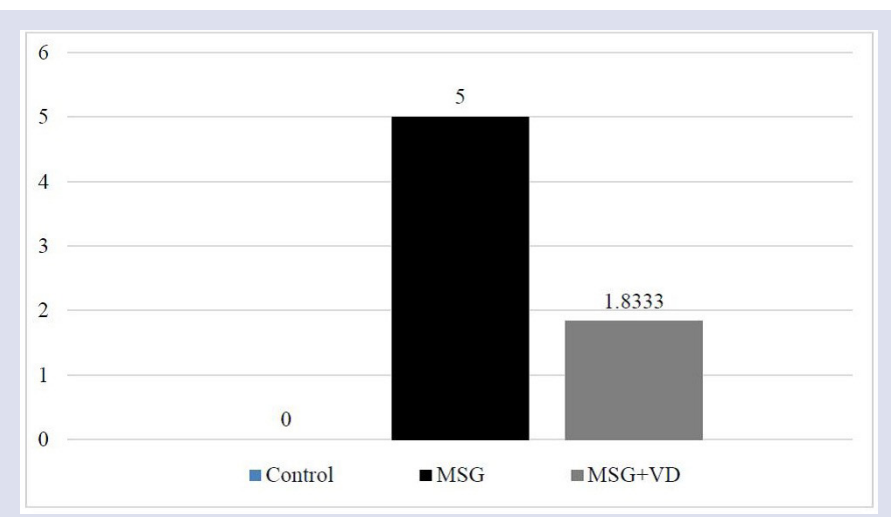

Figure 7: Average NASH Score, $p<0.001$ in between the experimental groups.

extent of steatosis was statistically significant, p-value being $<0.001$ [Figure 5]. Lobular inflammation was shown in all specimens of Group I, whereas only two specimens in the Group II showed inflammation. Liver cell ballooning, fibrosis and clear fatty changes were seen in three of the six specimens of Group I [Figure 3] whereas, Group II did not show any of these changes. Glycogenated nuclei were seen in four specimens of the rats treated with MSG when compared with Group II [Figure 6]. The score which is considered as diagnostic criteria for NASH was observed in the MSG treated group when compared with the other groups. Differences in NASH score between individual groups were statistically significant, p-value being $<0.001$ [Figure 7]

\section{DISCUSSION}

MSG is a distinct flavor that most of us have experienced most commonly in the tasty Chinese food treats and Asian food ingredients. MSG can cause severe health problems ranging from headaches to strokes in people of all age groups and both the sexes. However, extra precaution is required during the period of pregnancy as it may prove harmful to the mother as well as the fetus developing inside her womb. Glutamate exposure in pregnant women and children could thus possibly be linked higher incidence of toxicity manifestations which can be attributed to immature blood-brain barrier in younger age group. In the present study MSG induced hepatic damage was reversed in the vitamin D treated rats. The results of the present study show the ameliorative role of vitamin D in minimizing the MSG induced hepatic damage.

In the recent years, the incidence of NAFLD is increasing worldwide. Research shows that it is greatly influenced by the dietary factors. ${ }^{1}$ The histological changes in the neonatal liver tissues were identical with the non-alcoholic steatohepatitis. This was observed in the short duration of exposure when compared to much longer time of exposure in the adult rats. Based on this finding we suggest that MSG induced hepatic damage might provide a novel animal model of NASH. This makes gestational and lactational administration as used in our study a much quicker way to induce steatohepatitis.

Vitamin D deficiency is commonly associated with NAFLD and has even been correlated with disease severity. ${ }^{5}$ Vitamin D Receptor (VDR) present in the hepatocytes have shown to play role in glucose and lipid metabolism. ${ }^{8}$ The irreversible changes of fibrosis and glycogenation of nuclei were absent in vitamin D co-administered group implying that vitamin $\mathrm{D}$ might have a beneficial role in preventing progression of non-alcoholic steatohepatitis. Inflammatory amelioration of steatohepatitis by vitamin $\mathrm{D}$ could be explained by studies showing high levels of 
adiponectin, an anti-inflammatory agent found in higher levels in blood of subjects supplemented with vitamin D. ${ }^{9}$ Irreversible changes in fibrosis was seen in the animals treated with Vitamin. This could be due to the anti-fibrotic property of vitamin $\mathrm{D}$. This observation agrees with the previous findings. ${ }^{10,11}$ The finding of this study strengthens the hypothesis of a possible link between vitamin $\mathrm{D}$ and metabolic syndrome. ${ }^{5} \mathrm{Cumu}$ latively, our study reports suggest that vitamin $\mathrm{D}$ replacement might be effective in the treatment of NAFLD and also supplementation of diet rich in vitamin $\mathrm{D}$ might be helpful in minimizing the hepatic damage in the pregnant women consuming food containing MSG .However, clinical trials are required to better evaluate the efficacy of vitamin D replacement and parameters of therapy in NAFLD.

\section{IMPLICATIONS}

Vitamin D replacement might be effective in the treatment of NAFLD. Supplementation of diet rich in vitamin D might be helpful in minimizing the hepatic damage in the pregnant women who are consuming more food containing high levels of MSG.

\section{ACKNOWLEDGEMENT}

We are thankful to Manipal-University (MU), Karnataka, India, for supporting the Research work. We are also thankful to the Department of Physiology and Department of Pathology Kasturba Medical College (KMC), Mangalore, Manipal-University (MU), Karnataka, India, for providing the laboratory and necessary instruments for conducting the experiments.

\section{CONFLICT OF INTEREST}

No conflict of interest is declared.

\section{ABBREVATION}

MSG: Monosodium glutamate; NAFLD: Nonalcoholic Fatty Liver Disease; BW: Body weight; SD: Standard deviation; VDR: Vitamin D Receptor (VDR)

\section{REFERENCES}

1. Nakanishi $Y$, Tsuneyama $K$, Fujimoto $M$, Salunga $T L$, Nomoto $K$, An JL, et al. Monosodium glutamate (MSG): a villain and promoter of liver inflammation and dysplasia. J Autoimmun. 2008;30(1):42-50.

2. Mello MA, Souza CT, Braga LR, Dos-Santos JW, Ribeiro IA, Gobatto CA. Glucose tolerance and insulin action in monosodium glutamate (MSG) obese exercise-trained rats. Physiol Chem Phys Med NMR. 2001;33(1):63-71.

3. Matyšková R, Maletínská L, Maixnerová J, Pirník Z, Kiss A, Zelezná B. Comparison of the obesity phenotypes related to monosodium glutamate effect on arcuate nucleus and/or the high fat diet feeding in C57BI/6 and NMRI mice. Physiol Res. 2008;57(5):727-34

4. Larsen PJ, Mikkelsen JD, Jessop D, Lightman SL, Chowdrey HS. Neonatal monosodium glutamate treatment alters both the activity and the sensitivity of the rat hypothalamo-pituitary-adrenocortical axis. J Endocrinol. 1994;141(3):497-503.

5. Ju SY, Jeong HS, Kim do H. Blood vitamin D status and metabolic syndrome in the general adult population: a dose-response meta-analysis. J Clin Endocrinol Metab. 2013; 99(3):1053-63.

6. Pittas AG, Lau J, Hu FB, Dawson-Hughes B. The role of vitamin D and calcium in type 2 diabetes. A systematic review and meta-analysis. J Clin Endocrinol Metab. 2007; 92(6):2017-29

7. Kleiner DE, Brunt EM, Van-Natta M, Behling C, Contos MJ, Cummings OW, et al. Nonalcoholic Steatohepatitis Clinical Research Network: Design and validation of a histological scoring system for nonalcoholic fatty liver disease. Hepatology 2005; 41(6):1313-21.

8. Maestro B, Dávila N, Carranza MC, Calle C. Identification of a Vitamin D response element in the human insulin receptor gene promoter. J Steroid Biochem Mol Biol. 2003; 84(2):223-30.

9. Vaidya A, Williams JS, Forman JP. The independent association between 25-hydroxyvitamin $D$ and adiponectin and its relationship with $\mathrm{BMI}$ in two large cohorts: the NHS and the HPFS. Obesity. 2012;20(1):186-91.

10. Abramovitch S, Dahan-Bachar L, Sharvit E, Weisman Y, Ben-Tov A, Brazowski E, et al. Vitamin $D$ inhibits proliferation and profibrotic marker expression in hepatic stellate cells and decreases thioacetamide-induced liver fibrosis in rats. Gut. 2011;60(12):1728-37.

11. Zerr $\mathrm{P}$, Vollath $\mathrm{S}$, Palumbo-Zerr $\mathrm{K}$, Tomcik $\mathrm{M}$, Huang J, Distler $\mathrm{A}$, et al. Vitamin $D$ receptor regulates TGF- $\beta$ signalling in systemic sclerosis. Ann Rheum Dis. 2014;3(2):50-5.

12. Azak A, Huddam B, Haberal N, Koçak G, Ortabozkoyun L, Senes M, et al. Effect of novel vitamin $D$ receptor activator paricalcitol on renal ischaemia/reperfusion injury in rats. Ann R Coll Surg Engl. 2013;95(7):489-94.

\section{GRAPHICAL ABSTRACT}
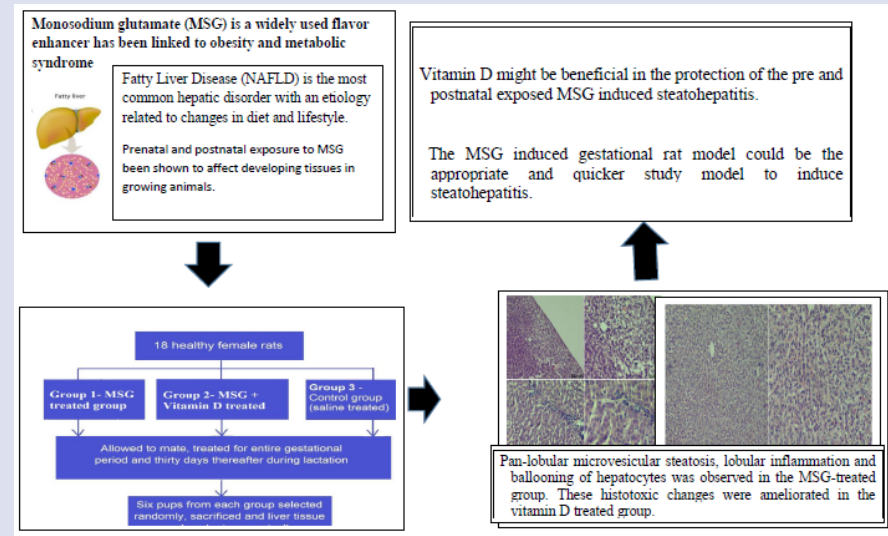

\section{SUMMARY}

- Administration of vitamin D successfully ameliorates steatohepatitis in animal models. Vitamin $D$ might be beneficial in the protection of the pre-and postnatal exposed MSG induced steatohepatitis. Further human clinical trials could establish the beneficial role of Vitamin D supplementation during pregnancy and lactation.

\section{ABOUT AUTHORS}

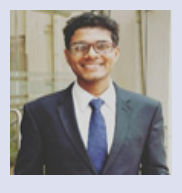

Mr. Nandan Padmanabha, is an undergraduate medical student currently in Final MBBS. He is the secretory of Manipal Academy of Higher Education Student Research Forum, Mangalore campus and treasurer at American Medical student association(AMSA), Mangalore Chapter. His areas of research include food toxicity and toxicity amelioration.

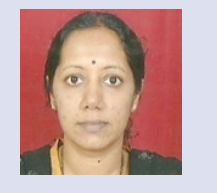

Dr. Nirupama M is an Associate Professor of, Kasturba Medical College Mangalore, Manipal Academy of Higher Education. She is also a PG guide and also guided ICMR projects. Her areas of interest include thyroid cytology, thyroid hematology. She has published many research papers in indexed journal. 


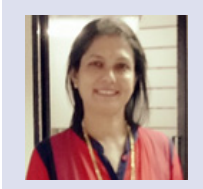

Dr. .Nayanatara Arun Kumar is currently working as an Associate Professor of Physiology, Kasturba Medical College Mangalore, Manipal Academy of Higher Education. She is a recognized Ph.D guide of Manipal Academy of Higher Education. Her research area is mainly focused on stress, Radioprotective effects of herbal extracts, neurophysiology, stress, food additives and alcohol. She has more than 80 research publications in indexed journals. She has been working as a Principal Investigator for BRNS funded project

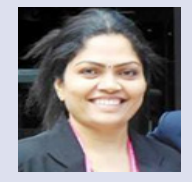

Dr Bhagyalakshmi K: Professor and Head, Department of Physiology, Kasturba Medical College, Mangalore, Manipal Academy of Higher Education. She received her MD (Doctor of Medicine) in Physiology at MAHE in the year 2005 and her MBBS (Bachelor of Medicine and Bachelor of Surgery) from Mysore University in 1999. Her research interest includes Heart rate variability, Yoga obesity and animal studies. She is the recognized Ph.D guide of Manipal Academy of Higher Education.

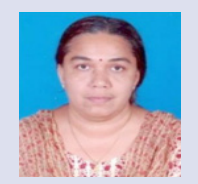

Dr. Jyothi R Kini is an Associate Professor of Pathology, Kasturba Medical College Mangalore, Manipal Academy of Higher Education. She is also a PG guide for MD students and Thesis. Her areas of interest include Onco pathology with focus on neurooncology and gastrointestinal pathology. She is incharge, hematology and clinical pathology in KMC hospital, Mangalore

Mr. Roopesh Poojary: Is a Research Scholar in Kasturba Medical College Mangalore, Manipal Academy of Higher Education, Karnataka, India. He has completed M.Sc. in the subject of Biotechnology at St. Aloysius College Mangalore. he is working as a Senior Research Fellow on the project funded by Board of Research in Nuclear Science Department of Atomic Energy, Government of India in the area of Radiation biology.

Cite this article: Padmanabha N, Nirupama M, Kumar NA, Bhagyalakshmi K, Kini JR, Poojary R. Ameliorative Role of Vitamin D on Prenatal and Postnatal Exposure of Monosodium Glutamate Induced Steatohepatitis in Rat Pups. Pharmacog J. 2018;10(2):371-5. 